\title{
Prognostic role of elevated mir-24-3p in breast cancer and its association with the metastatic process
}

\author{
Alireza Khodadadi-Jamayran ${ }^{1}$, Betul Akgol-Oksuz ${ }^{2}$, Yelena Afanasyeva ${ }^{3}$, Adriana \\ Heguy 4,5, Marae Thompson 6 , Karina Ray ${ }^{5}$, Ariadna Giro-Perafita6, Irma Sánchez ${ }^{4}$,

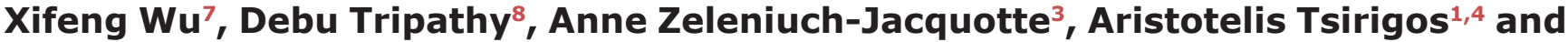 \\ Francisco J. Esteva ${ }^{6}$ \\ ${ }^{1}$ Applied Bioinformatics Laboratories, NYU School of Medicine, New York, NY, USA \\ ${ }^{2}$ Department Bioinformatics and Computational Biology, University of Massachusetts Medical School, Worcester, MA, USA \\ ${ }^{3}$ Division of Epidemiology, NYU School of Medicine, New York, NY, USA \\ ${ }^{4}$ Department of Pathology, NYU School of Medicine, New York, NY, USA \\ ${ }^{5}$ Genome Technology Center, NYU School of Medicine, New York, NY, USA \\ ${ }^{6}$ Division of Hematology/Oncology, Perlmutter Cancer Center, NYU Langone Health, New York, NY, USA \\ ${ }^{7}$ Department of Epidemiology, UT MD Anderson Cancer Center, Houston, TX, USA \\ ${ }^{8}$ Department of Breast Medical Oncology, UT MD Anderson Cancer Center, Houston, TX, USA \\ Correspondence to: Francisco J. Esteva, email: francisco.esteva@nyumc.org \\ Aristotelis Tsirigos, email: Aristotelis.Tsirigos@nyumc.org
}

Keywords: breast cancer; gene expression profiling

Received: July 08, $2017 \quad$ Accepted: January 13, $2018 \quad$ Published: February 05, 2018

Copyright: Khodadadi-Jamayran et al. This is an open-access article distributed under the terms of the Creative Commons Attribution License 3.0 (CC BY 3.0), which permits unrestricted use, distribution, and reproduction in any medium, provided the original author and source are credited.

\section{ABSTRACT}

MicroRNAs have been shown to play important roles in breast cancer progression and can serve as biomarkers. To assess the prognostic role of a panel of miRNAs in breast cancer, we collected plasma prospectively at the time of initial diagnosis from 1,780 patients with stage I-III breast cancer prior to definitive treatment. We identified plasma from 115 patients who subsequently developed distant metastases and 115 patients without metastatic disease. Both groups were matched by: age at blood collection, year of blood collection, breast cancer subtype, and stage. The median follow up was 3.4 years (range, 1-9 years). We extracted RNA from plasma and analyzed the expression of 800 miRNAs using Nanostring technology. We then assessed the expression of miRNAs in primary and metastatic breast cancer samples from The Cancer Genome Atlas (TCGA). We found that, miR-24-3p was upregulated in patients with metastases, both in plasma and in breast cancer tissues. Patients whose primary tumors expressed high levels of miR-24-3p had a significantly lower survival rate compared to patients with low mir-24-3p levels in the TCGA cohort $(n=1,024)$. RNA-Seq data of the samples with the highest miR-24-3p expression versus those with the lowest miR-24-3p in the TCGA cohort identified a specific gene expression signature for those tumors with high miR-24-3p. Possible target genes for miR-24$3 p$ were predicted based on gene expression and binding site, and their effects on cancer pathways were evaluated. Cancer, breast cancer and proteoglycans were the top three pathways affected by miR-24-3p overexpression. 


\section{INTRODUCTION}

Breast cancer is the most common malignancy in women, accounting for $30 \%$ of new invasive cancers expected to be diagnosed in the US in 2017 [1]. The most important prognostic factor is the stage at presentation, which is determined by the tumor size, the number of axillary lymph nodes involved and the presence/absence of overt metastases. Although the presence of distant metastases at diagnosis (stage IV) is the most ominous sign, women with stage I-III may also harbor occult metastases. It takes years from the initial transformation events of mammary cells to the detection of imaging/ clinical changes that result in a breast cancer diagnosis. During this time cancer cells can spread through the circulation to distant sites. The metastatic process is extremely complex and requires adaption mechanisms by cancer cells including but not limited to migration, invasion, penetration into blood vessels, circulation through the bloodstream, extravasation and expansion in distant organs [2]. However, the regulation of the metastatic process at cellular and molecular levels is not well defined for individual patients. Molecular markers that are commonly used in clinical practice include the estrogen receptor (ER), progesterone receptor (PR), the human epidermal growth factor receptor 2 (HER2), the $\mathrm{Ki}-67$ proliferation marker and multi-gene assays [35]. These prognostic and predictive factors are used to estimate the risk of recurrence and the potential benefit associated with systemic therapies, including HER2targeted therapy, endocrine therapy and chemotherapy [68]. Despite improvements in local, regional and systemic therapies for breast cancer, 40,610 women are expected to die from metastatic breast cancer in the US in 2017 [1]. Therefore, there is a need to identify novel prognostic and predictive markers of occult metastases to better individualize adjuvant systemic therapy and develop new therapeutic approaches to eradicate occult metastases in women with early-stage breast cancer $[9,10]$.

MicroRNAs (miRNAs) are small, noncoding RNA molecules 20-23 nucleotides in length that play key roles in regulation of cell division, differentiation, and death [11]. One of the main functions of miRNAs is to silence gene expression by binding to complementary sequences in the 3'UTR of target mRNAs [12]. Dysregulation of miRNA expression has been linked to carcinogenesis, invasion and metastasis [13]. MicroRNAs can be detected and quantified reliably in plasma and tissue from cancer patients and have potential as biomarkers [14, 15]. Previous studies revealed correlations between miRNA detection and prognosis in breast cancer. However, results have not been consistent or reproducible across studies in part because of the different populations evaluated and the methods used to identify miRNAs. Furthermore, there are limited data regarding molecular mechanisms for single
miRNAs or miRNA signatures that may impact prognosis in breast cancer patients.

We conducted a study to determine the prognostic role of circulating plasma miRNAs in patients with earlystage breast cancer. We measured the expression of 800 miRNAs in plasma we collected prospectively at the time of diagnosis, before definitive primary treatment. We detected miRNAs that were expressed at higher levels in patients with stage I-III who subsequently developed metastatic disease. We then evaluated the differential expression of miRNAs in primary breast cancer tissue using The Cancer Genome Atlas (TCGA) database. Both analyses revealed mir-24-3p as a top candidate for further evaluation as a biomarker for breast cancer metastasis.

\section{RESULTS}

\section{Plasma microRNA transcriptome analysis identifies 29 differentially expressed microRNA in patients with early-stage breast cancer who subsequently developed metastasis}

Using Nanostring technology we profiled the microRNA transcriptome in plasma from 115 cases and 115 controls, respectively. In total, we obtained expression levels of $\sim 800$ microRNAs. To identify potentially prognostic microRNAs, we performed differential expression analysis between the two patient groups using DEseq2. We identified 29 differentially expressed microRNAs in patients who developed metastasis compared to patients who did not: 24 microRNAs were upregulated while 5 were downregulated (Figure 1A). A comprehensive list of the significantly differentially expressed microRNAs is shown in Table 1.

\section{mir-24-3p overexpression correlates with advanced stage, and triple negative/basal subtype in primary breast cancer tissues}

Motivated by the discovery of differentially expressed microRNAs in the plasma of metastatic versus nonmetastatic patients, we then hypothesized that some of the microRNA transcripts we detected in the plasma may have originated in the primary tumors either via circulating tumor cell parts, microvesicles or via exosomes. To find supporting evidence for our hypothesis, we analyzed microRNA expression data obtained from TCGA. We then performed differential microRNA expression analysis between stage I and stage IV patients. As demonstrated in the volcano plot in Figure 1B, eleven microRNAs (out of a total of 1881 microRNAs) were significantly upregulated in primary tumors of stage IV versus stage I patients. Interestingly, mir-24-3p was also found to be upregulated in the plasma samples from metastatic versus non-metastatic patients in our cohort. Kaplan-Meier survival analysis 
shows significantly lower survival rates ( $p$-value $=0.0024$, quantile-based log-rank test) in patients with elevated levels of mir-24-3p in primary tumors from TCGA (Figure 1C).

For TCGA data, Multivariate Analysis of Variance (MANOVA) indicated the association of mir-24-3p expression with known prognostic factors, i.e. subtype, stage and survival time ( $p$-value $=3.749 \mathrm{e}-12$ ). Univariate Analysis of Variance (ANOVA and $t$-test) has been performed on tumor subtypes and the results indicate showing a significant ( $\mathrm{p}$-value $<0.001$ ) difference between different subtypes (Figure 1D). We also examined the association of mir-24-3p survival with subtype and stage found a positive association in univariate analysis $(p=0.009)$ that remained statistically significant after adjusting for intrinsic subtype and stage $(\mathrm{p}=0.004)$ (Supplementary Figure 1, right panel (B)). A similar trend was observed in the Nanostring plasma dataset (Supplementary Figure 1, left panel (A)), however it was marginally significant, probably due to the limited number of patients and lower levels of mir-24-3p in plasma compared to tumor tissue. In our patient cohort, there was no difference in mean mir-24-3p between grade I and grade II tumors, but the mean mir-24-3p expression in grade III tumors was higher than in grade I tumors $(\mathrm{p}=0.04)$. Grade was not available for TCGA (Supplementary Figure 1).
A
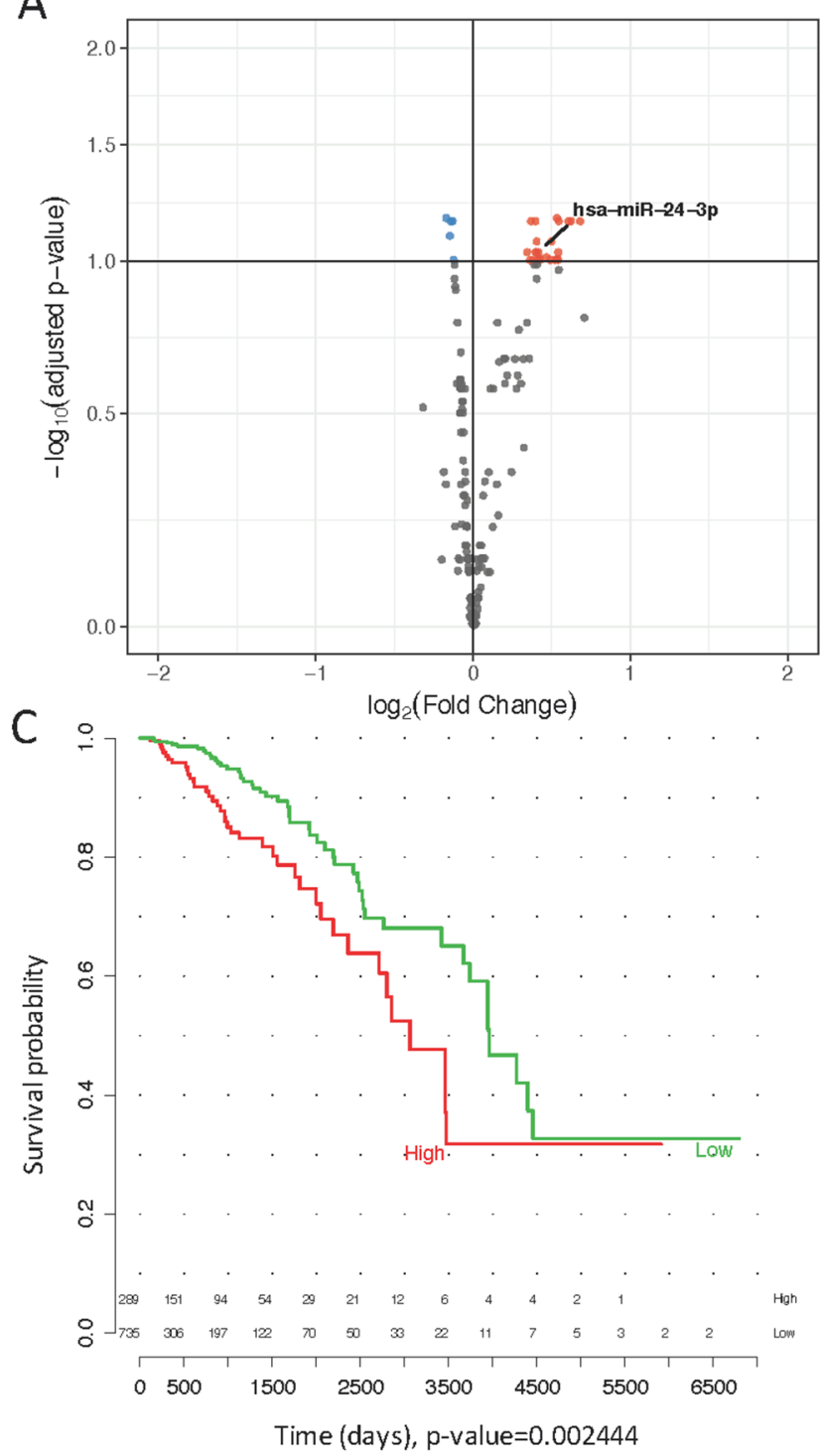

B
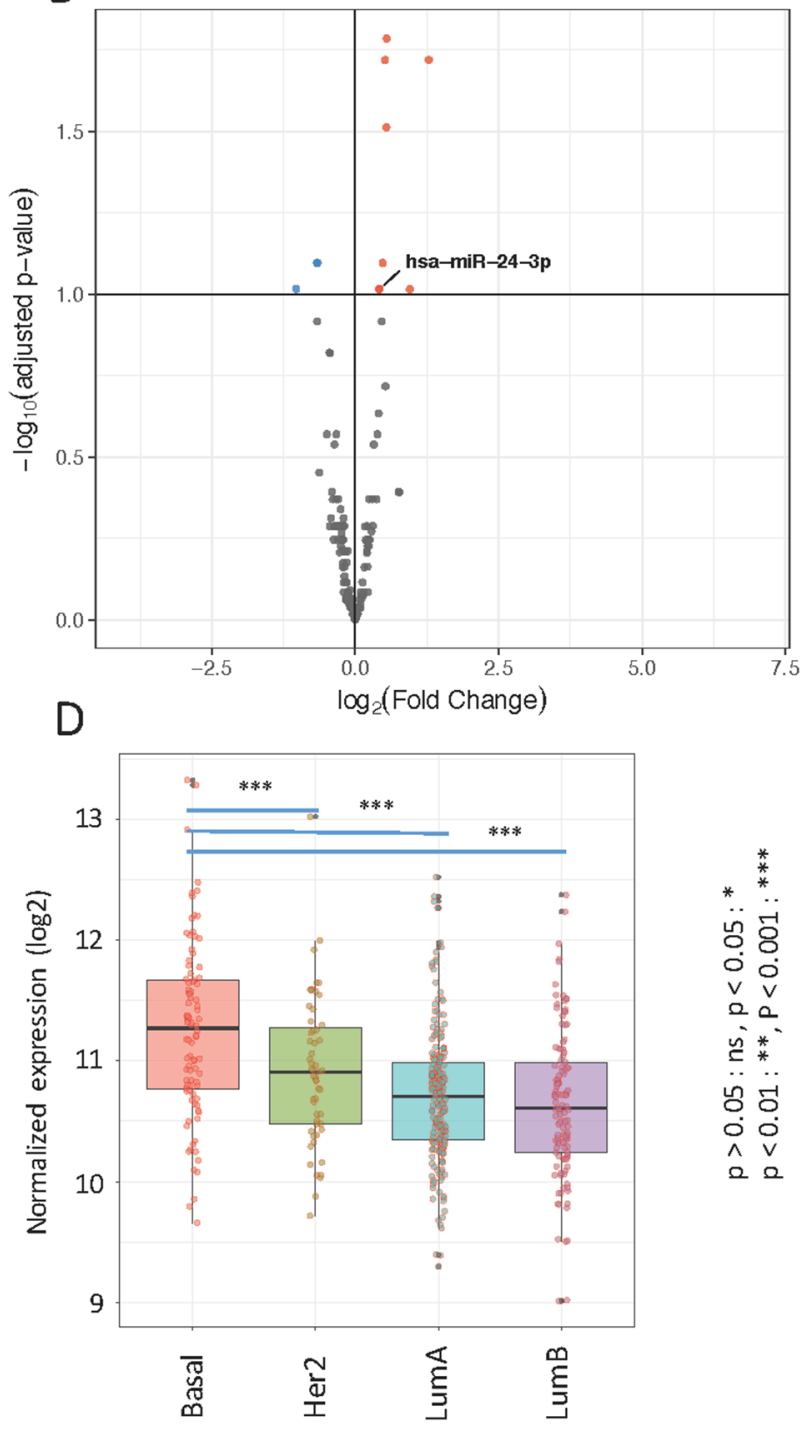

Figure 1: (A) Volcano plot of differentially expressed miRNAs in metastatic and non-metastatic serum samples obtained by Nanostring data analysis. Upregulated miRNAs are plotted in red and downregulated in blue (B) Volcano plot of differentially expressed miRNAs in TCGA stage I versus stage IV primary tumor samples. Upregulated miRNAs in Stage IV are plotted in red and downregulated in blue (C) Survival curve of mir-24-3p in TCGA patients (D). Distribution of mir-24-3p across different tumor subtypes in TCGA primary tumors. 
Table 1: List of the significantly differentially expressed microRNAs in plasma of metastatic vs. non-metastatic patients

\begin{tabular}{|c|c|c|c|c|c|}
\hline Gene_name & Control_mean & Metastasis_mean & foldChange & pvalue & padj \\
\hline hsa-miR-126-3p & 421.9134289 & 676.8186276 & 1.604164697 & 0.002945286 & 0.069244359 \\
\hline hsa-miR-130a-3p & 82.35289258 & 126.8828971 & 1.540721803 & 0.003637321 & 0.069244359 \\
\hline hsa-miR-181a-5p & 26.338105 & 40.11759065 & 1.523176806 & 0.001810582 & 0.069244359 \\
\hline hsa-miR-106a-5p+hsa-miR-17-5p & 42.32371603 & 61.78223478 & 1.459754496 & 0.004196628 & 0.069244359 \\
\hline hsa-miR-15a-5p & 57.75558022 & 84.09167839 & 1.455992271 & 0.010034894 & 0.091986529 \\
\hline hsa-miR-142-3p & 307.0759047 & 447.0948109 & 1.455974904 & 0.015969554 & 0.098567251 \\
\hline hsa-let-7g-5p & 85.84623264 & 124.5787981 & 1.451185384 & 0.012432581 & 0.097684567 \\
\hline hsa-miR-146a-5p & 25.87165231 & 37.48361606 & 1.448829615 & 0.000658213 & 0.067224967 \\
\hline hsa-miR-15b-5p & 113.2087794 & 162.6198738 & 1.436459917 & 0.016786562 & 0.098567251 \\
\hline hsa-miR-199a-5p & 34.088818 & 48.15377871 & 1.412597489 & 0.006576129 & 0.083466246 \\
\hline hsa-miR-191-5p & 114.5181748 & 161.0017431 & 1.405905598 & 0.017323941 & 0.098567251 \\
\hline hsa-miR-27b-3p & 17.42951055 & 24.09008977 & 1.38214379 & 0.011079524 & 0.096216921 \\
\hline hsa-miR-20a-5p+hsa-miR-20b-5p & 45.1 & 53392 & 1.341990447 & 0.014262422 & 0.098567251 \\
\hline hsa-let-7d-5p & 22.86627562 & 30.65721483 & 1.340717454 & 0.012421539 & 0.097684567 \\
\hline hsa-miR-24-3p & 16.88318331 & 22.53860549 & 1.334973689 & 0.009161539 & 0.091986529 \\
\hline hsa-miR-19b-3p & 27.72094601 & 36.77714893 & 1.326691698 & 0.014876929 & 0.098567251 \\
\hline hsa-miR-106b-5p & 16.56919141 & 21.93428224 & 1.323799194 & 0.006447937 & 0.083466246 \\
\hline hsa-miR-98-5p & 14.76934433 & 19.54707704 & 1.323489831 & 0.009004442 & 0.091986529 \\
\hline hsa-let-7i-5p & 49.96965086 & 65.92391001 & 1.31927898 & 0.008677135 & 0.091986529 \\
\hline hsa-miR-185-5p & 19.81740991 & 26.10801207 & 1.317428069 & 0.002642304 & 0.069244359 \\
\hline hsa-miR-151a-3p & 18.06574804 & 23.53997512 & 1.303016906 & 0.016270605 & 0.098567251 \\
\hline hsa-miR-92a-3p & 18.98911204 & 24.51292998 & 1.290893957 & 0.003233125 & 0.069244359 \\
\hline hsa-miR-361-5p & 14.98231138 & 19.2511097 & 1.284922547 & 0.016185416 & 0.098567251 \\
\hline hsa-miR-32-5p & 21.20346146 & 26.94874863 & 1.27095987 & 0.009784947 & 0.091986529 \\
\hline hsa-miR-516a-3p+hsa-miR-516b-3p & 17.43257585 & 16.02094928 & -1.088111294 & 0.013722192 & 0.098567251 \\
\hline hsa-miR-518f-3p & 20.49794068 & 18.74217241 & -1.093680083 & 0.002215359 & 0.069244359 \\
\hline hsa-miR-125b-5p & 19.52071838 & 17.705905 & -1.102497635 & 0.00400355 & 0.069244359 \\
\hline hsa-miR-1293 & 16.4858169 & 14.89180043 & -1.107039876 & 0.005290175 & 0.07935262 \\
\hline hsa-miR-1236-3p & 17.64853298 & 15.68462151 & -1.125212552 & 0.000814848 & 0.067224967 \\
\hline
\end{tabular}

\section{mir-24-3p is associated with pathways upregulated in cancer}

Because of the significant correlation of mir24-3p expression levels with patient survival rates, we further investigated its potential regulatory role in primary tumors. We sorted the TCGA samples based on their mir-24-3p expression and took the top 25 and the bottom 25 samples to perform differential expression analysis on those conditions or two patients groups
(Figure 2A). Dimensionality reduction of protein-coding gene expression profiles of the two patient groups using t-distributed stochastic neighbor embedding [16] revealed a good separation of the two patient groups (Figure 2B), suggesting that tumors with high mir-24-3p expression are dependent on transcriptional programs that are distinct from the ones active in patients with low mir-24-3p expression. To identify these transcriptional programs, we first performed differential expression analysis of the protein-coding genes (TCGA RNA-Seq data) between the 
two patient groups. This analysis yielded a large number of differentially expressed genes (2128 upregulated and 1190 downregulated), highlighted in the volcano plot in Figure 2C. The top 500 significantly differentially expressed genes are depicted using an expression heatmap representation in Figure 2D. Pathway analysis of these protein-coding genes identified several pathways involved in cancer. Top three pathways includes pathways in cancer (KEGG: has05200), proteoglycans in cancer (KEGG: 05205) and breast cancer (KEGG: hsa05224) (Figure 3A). A detailed overview of all the significantly (FDR $<0.1)$ differentially expressed genes in pathways in cancer (KEGG: has05200 are shown in Figure 3B. Similar pathway overviews were obtained for breast cancer (Supplementary Figure 2) and proteoglycans in cancer (Supplementary Figure 3).

\section{mir-24-3p protein-coding target genes are involved in cancer pathways}

We identified 746 putative protein coding target genes for mir-24-3p using TargetScan (http://www. targetscan.org). We then examined whether mir-24-3p putative targets are significantly differentially expressed in TCGA samples with high mir-24-3p compared to samples with low mir-24-3p expression. Of the 745 genes, 316
A

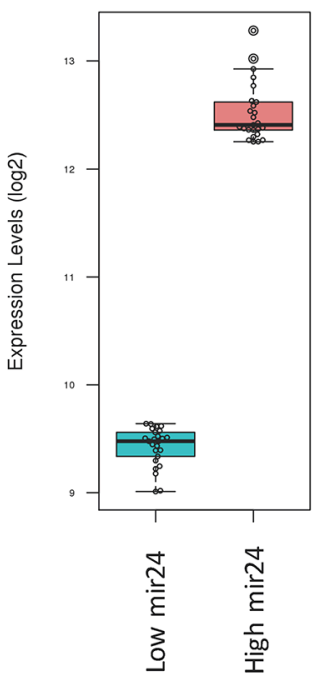

C

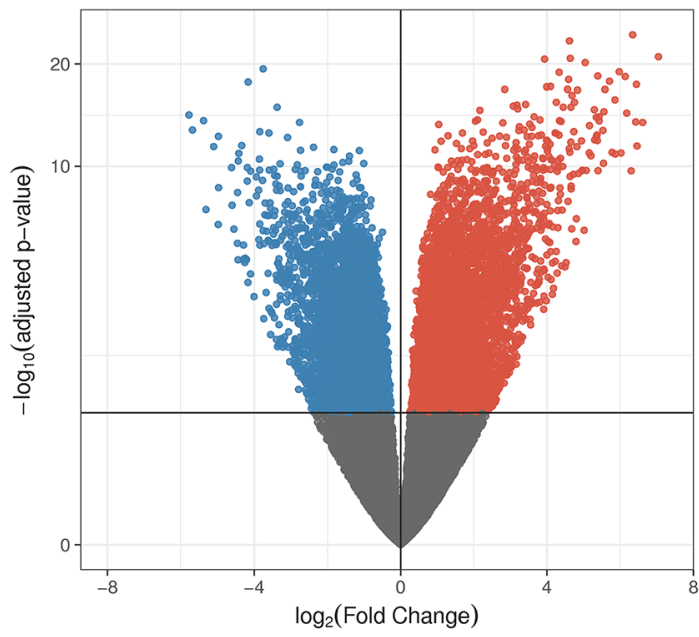

B

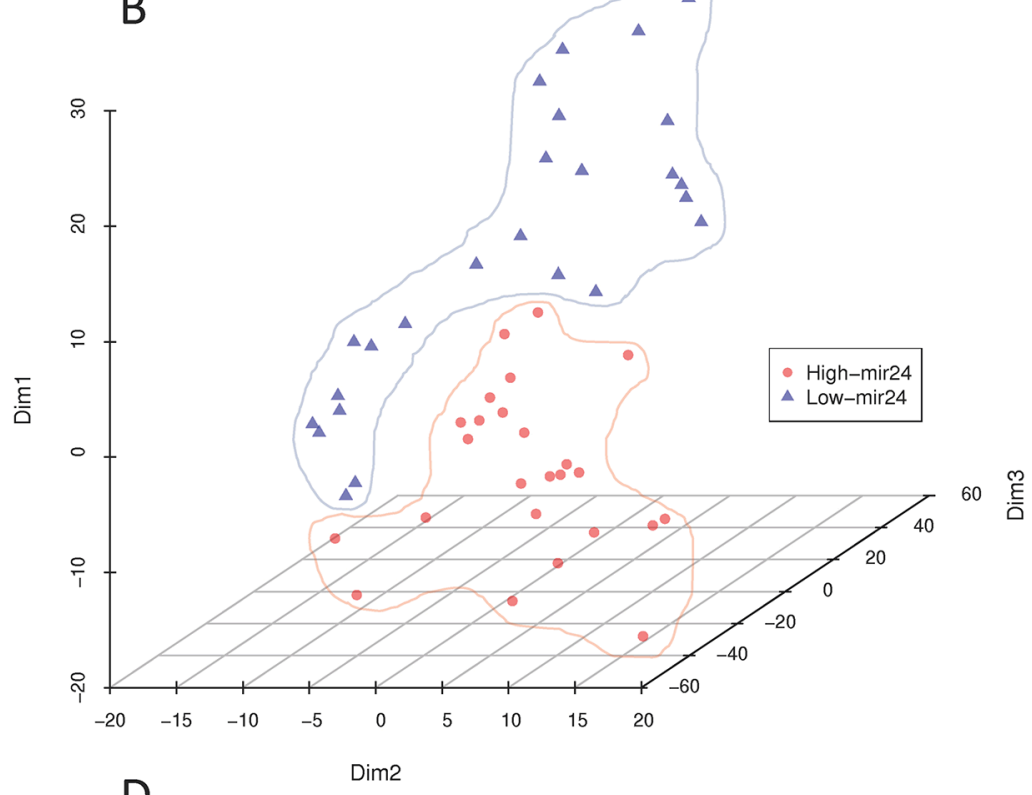

Low mir24

High mir24

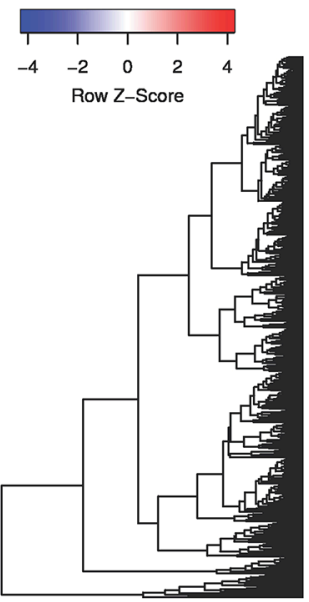

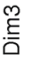
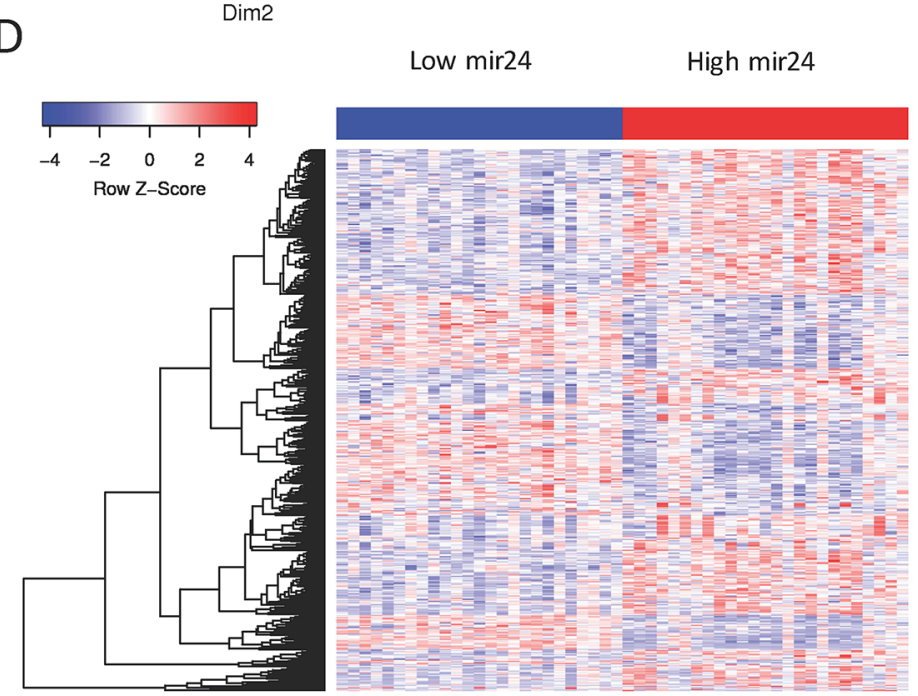

Figure 2: (A) Box-plot of mir-24-3p levels in patients showing the highest $(n=25)$ and lowest $(n=25)$ expressing levels of mir-24-3p in primary tumors from the TCGA (B) t-SNE plot of patients with high and low mir-24-3p expressing levels showing the separation of the two conditions (C) Volcano plot of the genes differentially expressed based on mir-24-3p expression in primary tumors (high versus low) (D) Heatmap of the top 500 genes differentially expressed in patients with high versus low mir-24-3p levels. 


\section{Cases}

Controls

$\mathrm{n}=\mathbf{1 1 5}$

$\mathrm{n}=\mathbf{1 1 5}$

Age at diagnosis, years

Median (range)

$48(22,83)$

$48(24,88)$

Race/ethnicity

Caucasian

$66(57 \%)$

$69(60 \%)$

African-American

$25(22 \%)$

$16(14 \%)$

Asian/Pacific Islanders

$5(4 \%)$

$6(5 \%)$

Hispanic

$18(16 \%)$

$23(20 \%)$

Other

$1(1 \%)$

$1(1 \%)$

Menopausal status at diagnosis

Premenopausal

$54(47 \%)$

$58(51 \%)$

Perimenopausal

$4(3 \%)$

$9(8 \%)$

Postmenopausal

$57(50 \%)$

$48(41 \%)$

Stage at sample collection

I

II

$4(3 \%)$

$4(3 \%)$

$49(43 \%)$

$55(48 \%)$

III

$62(54 \%)$

$56(49 \%)$

Subtype based on ER, PR, HER2

ER/PR+ and HER2-

$57(50 \%)$

$58(50 \%)$

HER2+

$19(16 \%)$

$18(16 \%)$

Triple negative

$39(34 \%)$

$39(34 \%)$

Year of sampling

2003
2004
2005
2006
2007
2008
2009

$12(10 \%)$

$8(7 \%)$

$14(12 \%)$

$22(19 \%)$

$23(20 \%)$

$17(15 \%)$

$16(14 \%)$

$14(12 \%)$

$12(10 \%)$

$17(15 \%)$

$27(23 \%)$

$27(23 \%)$

$11(10 \%)$

$10(9 \%)$

Time to metastases, years

Median (range)

$2.3(0.6,9.0)$

genes were significantly differentially expressed (FDR < 0.1 ). Of these, 134 genes were down-regulated and 182 genes were up-regulated when mir-24-3p levels were high (Figure 4A). To identify the most stringent list of putative targets, we additionally filtered the 316 genes based on fold change and identified 27 genes significantly downregulated by more than 2 -fold in patients with high levels of mir-24-3p (Figure 4B). A pathway analysis of all the target genes revealed that mir-24-3p may significantly regulate genes involved in cancer ( $p$-value $<0.05)$ (Supplementary Figure 4).

\section{DISCUSSION}

Using nanostring and RNA-sequencing technologies we identified miR-24-3p as a potential 
novel marker of breast cancer metastases in breast cancer. MiR-24-3p was expressed at high levels in the plasma from early-stage breast cancer patients destined to metastasize despite best available therapy, and in primary breast cancer tissue from patients who presented with stage IV at the time of diagnosis. High expression of mir-24-3p in primary breast cancer tissues correlated with a poor survival rate in TCGA. These data strongly suggest mir-24-3p is involved in the metastatic process in breast cancer patients.

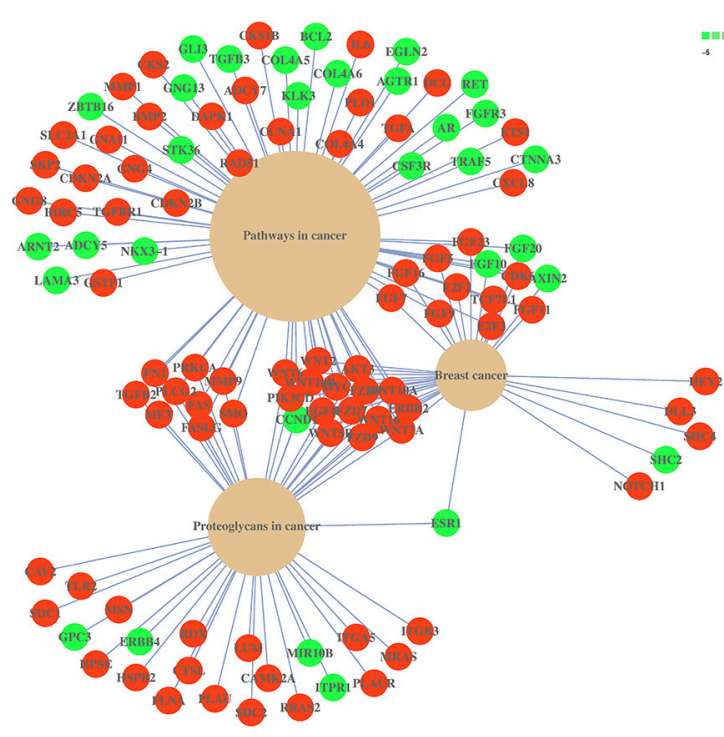

B

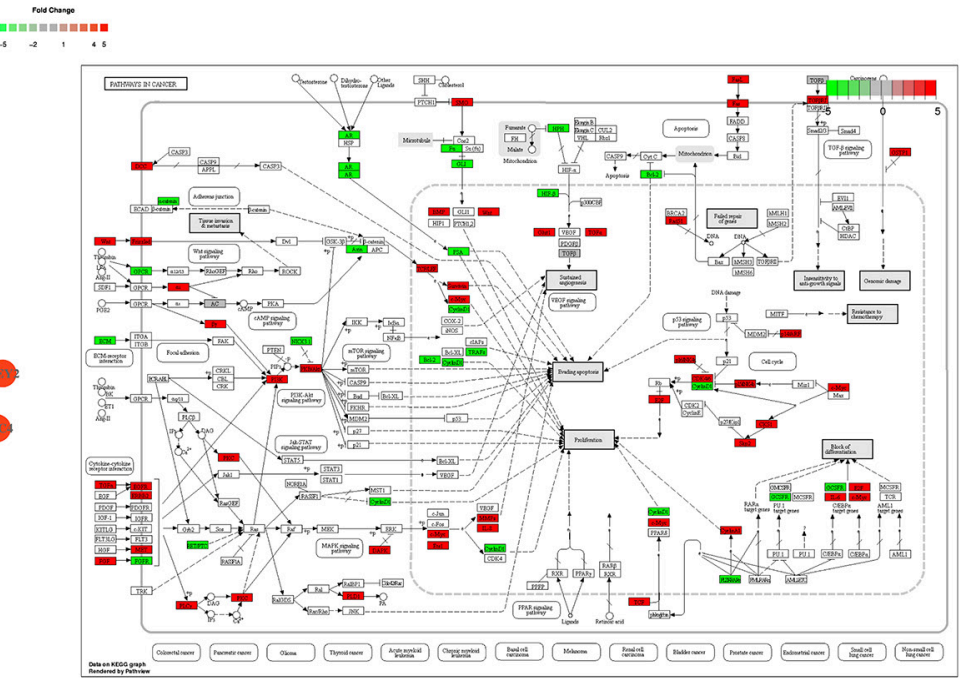

Figure 3: (A) The top three significantly $(\mathrm{P}$ value $<0.05)$ effected pathways in patients with high mir-24-3p vs. low mir-24-3p ( $\mathrm{n}=50)$ (B) Heat map overview of the principal pathways involved in cancer (KEGG: hsa05200). Upregulated genes are in red and downregulated genes are shown in green.

A

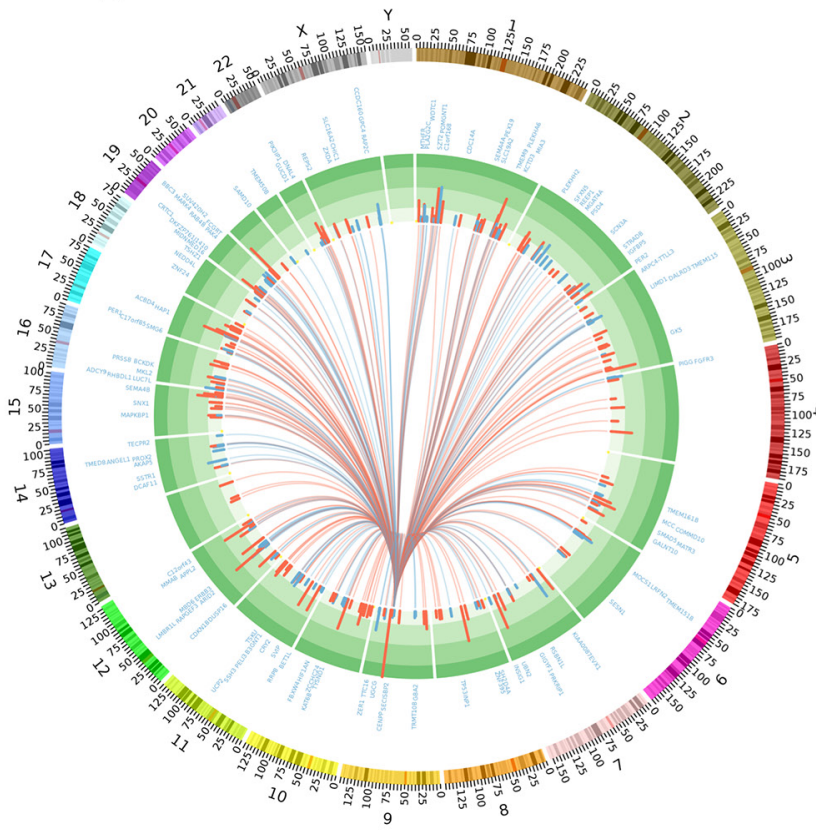

B

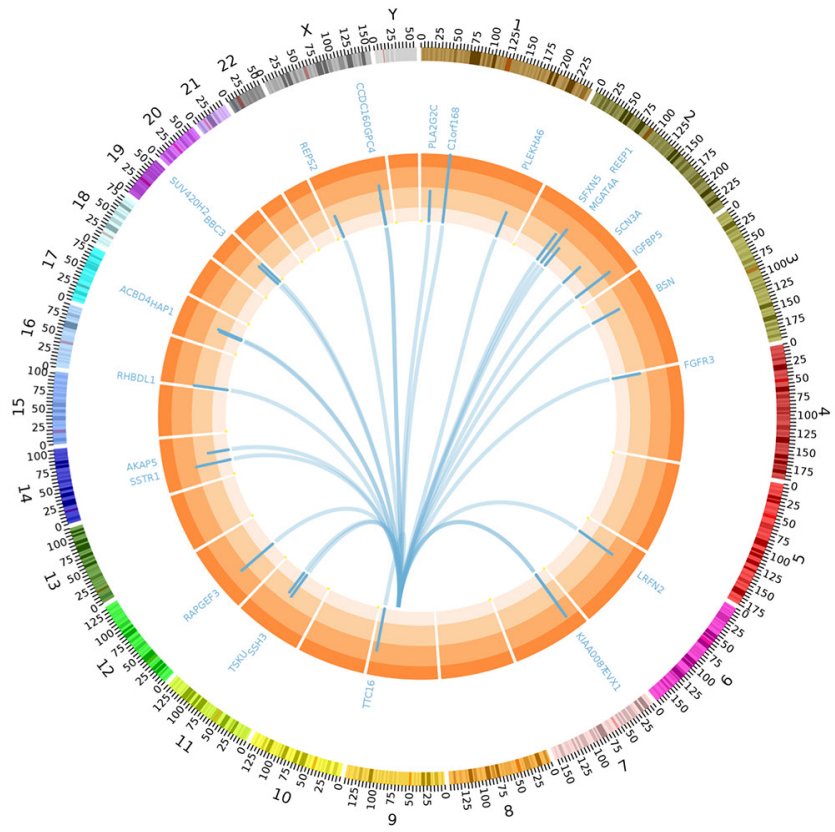

Figure 4: Circos plots depicting (A) 316 deferentially expressed genes (FDR $<0.1$ ) out of 745 target genes detected by TargetScan for mir-24-3p (differentially expressed in patients with high mir-24-3p levels vs. low miR-24). Red arcs show genes upregulated and blue downregulated in samples with high mir-24-3p and the histogram represents the fold change. (B) 27 significantly down regulated genes (FDR $<0.1 \&$ fold change $>2$ ) out of 745 target genes detected by TargetScan for mir-24-3p showing the most possible targets. 
There is great interest in the development of liquid biopsies using next generation sequencing. While most studies are focussed on the detection of mutations at the DNA level, Nanostring and RNA-Seq technologies allow detection of nucleic acids in plasma and tissues with high sensitivity and specificity. The demonstration that miRNAs are stable and can be extracted from tissues, blood, and other body fluid without degradation makes them ideal biomarkers for diagnosis and prognosis in breast cancer and other solid tumors. A meta-analysis of studies designed to determine the prognostic role of plasma miRNAs in patients with triple negative breast cancer showed a correlation between high circulating miRNA expression and lower disease-free survival, relapse-free survival and distant metastasis-free survival rates [17]. Sochor and collaborators [18] found that high-risk patients (classified as TNBC, HER2, highly proliferative or with positive node involvement) expressed higher levels in plasma of miRNAs related to cancer (oncomiRs), including miR-2. Several studies have identified mir-24-3p as a potential oncomiR in breast cancer, as its expression is specifically upregulated in both tumor and plasma from patients that developed the disease compare to healthy controls [19].

RNA-Seq data reported by Fiskaa, et al [20] and Hannafon, et al [21] showed mir-24-3p is released from the cells in exosomes. We re-analyzed the data in both studies and found that mir-24-3p is significantly upregulated in breast cancer cell lines versus non-breast cancer cell lines (p-value $<0.05$ using a t-test). (data not shown).

In addition to the strong correlation between plasma levels of mir-24-3p and the probability of developing metastasis in patients with early-stage of breast cancer, we identified a specific gene expression signature in patients with high levels of mir-24-3p in primary tumors. Interestingly, differential gene expression analysis showed that the principal genes deregulated are affecting the main pathways related to cancer, such as survival, migration and proliferation, pathways directly related to the process of metastasis.

Many studies have linked the expression of mir24-3p with breast cancer progression and metastasis by regulating genes and signaling pathways associated with cell cycle progression [19], DNA repair and drug resistance [22]. mir-24-3p directly targets p27(Kip1) and $\mathrm{p} 16(\mathrm{Ink} 4 \mathrm{a})$ in primary keratinocyte and in different cancer derived cell lines promoting their proliferation, suggesting that mir-24-3p is involved in carcinogenesis by post-transcriptional regulation of cyclin-dependent kinase [23]. Du et al. observed that mir-24-3p was upregulated in breast cancer tissues compared to benign tissues in a small cohort of patients. In vitro and in vivo studies indicated that the expression of mir-24$3 p$ enhanced tumor growth, invasion into local tissues, metastasis to lung tissues and decreased overall mouse survival by direct targeting PTPNO and PTPRF and therefore downregulating phosphorylation levels of EGFR [24]. In another study, the authors reported that mir-24-3p expression was significantly increased in HCC metastatic tumor tissues compared with matched nonmetastatic tumor tissues. These authors showed that mir24-3p could down-regulate $\mathrm{p} 53$ through binding to the 3 '-UTR of p53 mRNA, resulting in enhanced invasion in HCC cell lines [25]. Additionally, a recent report provides compelling data in support for a direct role of mir-24-3p in promoting breast tumor cell growth and metastasis in a xenograft mouse model via the regulation of ING5 [26]. Altogether, our results suggest that the overexpression of mir-24-3p during early-stages of breast cancer could drive gene-expression reprogramming to a more metastatic phenotype in breast cancer, and that the levels in plasma of mir-24-3p could be used as a feasible biomarker for patient prognosis and therefore help decision-making for treatment options.

In summary, we have identified mir-24-3p as a potential plasma biomarker of occult metastasis in patients with stage I-III breast cancer. Furthermore, we found that mir-24-3p is highly expressed in metastatic breast cancer tissue compared to primary breast cancer tissue, and that those tumors show specific gene expression signature. Our data show mir-24-3p plays an important role in regulation of the metastatic process in breast cancer, and justifies prospective studies to confirm the role of mir-24-3p and its gene targets as novel prognostic and predictive markers.

\section{MATERIALS AND METHODS}

\section{Study subjects}

A whole blood sample was collected prospectively at The University of Texas MD Anderson Cancer Center from 1,780 patients with stage I-III breast cancer, from 6/18/03 to 2/20/2012 after obtaining Institutional Review Board approval and written informed consent from participants. The blood sample was collected after initial diagnosis and prior to definitive treatment, which included either primary surgery or neoadjuvant chemotherapy depending on the clinical stage. All patients were treated uniformly with standard local and systemic therapies. Blood samples were centrifuged at $x 1200 \mathrm{~g}$ for 10 minutes at $4{ }^{\circ} \mathrm{C}$ to separate the blood cells, and the supernatant was transferred into microcentrifuge tubes and then centrifuged a second time at $\mathrm{x} 12,000 \mathrm{~g}$ for 10 minutes at $4{ }^{\circ} \mathrm{C}$ to completely remove the cellular components. Plasma was aliquoted and stored at $-80^{\circ} \mathrm{C}$ until use. Blood samples were processed and plasma was frozen within 4 hours of collection. Only patients recruited from 2003 to 2009 were used in the present study to ensure sufficient follow-up. At a median follow up of 3.4 years (range, 1-9 years), 119 patients developed distant metastases. Each of these patients was matched to a patient who was alive and 
not diagnosed with metastases at follow-up time equal or greater to that at which the case was diagnosed with her first metastasis (control). The matching factors were age (+/- 5 years) at blood collection, year of blood collection, breast cancer subtype, and stage. Out of total 119 cases, 4 cases were excluded because no eligible controls were found. As a result, 115 cases and 115 matched controls are included in the analysis. A summary of the patient characteristics is shown in Table 2.

\section{RNA extraction}

Total RNA was extracted from plasma using the Plasma/Serum RNA Purification Midi Kit (Norgen Biotek Corp.) according to the manufacturer's instructions. Spike in oligos were added as an internal control following the final lysis step according to the manufacturer's instructions. Eluted RNA volume was brought up to $420 \mu$ l using nuclease free water and passed through a pre-wet Amicon Ultra- 0.5 Centrifugal Filter Device (EMD Millipore). Diluted RNA was spun for 80 minutes at $14,000 \mathrm{xg}$ at room temperature and then for 2 minutes at $8,000 \mathrm{xg}$ to collect concentrated RNA, at a final volume of $20-25 \mu 1$.

\section{Nanostring for assessment of microRNA expression}

RNA quality and quantity were analyzed on an agilent Bioanalyzer 2100 using a pico chip. miRNA samples were hybridized using the Nanostring nCounter ( ) Human v3 miRNA Expression Assay, which includes 800 microRNAs, according to the manufacturer's protocol. $3 \mathrm{uL}$ of concentrated miRNA were used per sample. Hybridizations were processed on the nCounter Prep Station, and prepped cartridges were read on the Nanostring Digital Analyzer using 280 field of view counts. Data was analyzed with Nanostring nSolver 2.0. All samples were analyzed together using the default quality control measures and normalized to spike-ins added to samples before extraction.

\section{Computational analysis}

Raw nanostring data is preprocessed using Nanostring nSolver 2.0. First, the background threshold is determined, either in probe specific or more global manner. Then, the background is subtracted to determine the true counts. This subtraction generally improves downstream data analysis, such as fold-change estimation. The background subtracted nanostring data is then corrected for library sizes and differential expression analysis is performed using DESeq2. The read count tables for all the BRCA miRNA illumine sequencing samples (1207 samples) in TCGA were downloaded from The National Cancer Institute's (NCI) Genomic Data Commons (GDC). Of these samples, primary solid tumor samples $(\mathrm{N}=1047$, with complete clinical data annotation for cancer stage) and normal tissue samples $(\mathrm{N}=104)$ were used in this study. All the read count tables were then corrected for their library size differences based on their geometric library size factors using the DESeq2 package (v3.0) [27]; differential expression (DE) analysis was performed using this package. The PCA plots were visualized using ggplot2 package and the $\mathrm{R}$ 'dist' function was used to calculate the sample distances by setting the method as Euclidean. Euclidian distance heat-maps were visualized using ggplot2's "heatmap.2" function. mir-24-3p expression were used for survival analysis using Kaplan-Meier curves $(n=1024)$. Log-rank test was used to test for the survival distributions amongst our sample groups. The mean of miR24 expression was used as a threshold for low and high limits and the survival objects were calculated using the rms R package. Pathway analysis was performed using the clusterprofiler package [28]. We used TargetScan for miRNA target prediction. The Circo plots were generated using CIRCOS [29].

\section{Statistical analysis}

For both data sets, wald test $\mathrm{p}$ values and adjusted $\mathrm{p}$ values provided by DESeq2 package were used for differential expression analysis (adjusted $\mathrm{p}<0.1$ ) which is based on estimating dispersions and uses a negative binomial generalized linear model. For sample clustering, we performed a classical multidimensional scaling or Principal Component Analysis (PCA), $\mathrm{t}$-distributed stochastic neighbor embedding ( $\mathrm{t}-\mathrm{SNE}$ ) and a Euclidean distance based clustering. We used ANOVA and MANOVA to examine the association of mir-24-3p with known prognostic factors, i.e. subtype, grade, stage and survival time. For pathway and enrichment analysis, we used hypergeometric distribution tests performed by clusterProfiler package (adjusted $\mathrm{p}<0.1$ ). The data in both data sets were individually and comprehensively analyzed and visualized all in the R statistical environment (v3.2.5). The Cox proportional hazards model was used to assess the association of subtype, stage, and mir-24-3p with survival in the TCGA dataset. The log-rank test was also used for testing the survival rates and distributions.

\section{Statement of significance}

We identified mir-24-3p as a novel marker of occult metastases in plasma from patients with stage I-III breast cancer using nanostring technology. RNA-Seq data from The Cancer Genome Atlas showed association of mir-24$3 \mathrm{p}$ expression and metastases in primary breast cancer tissue. Expression of mir-24-3p was associated with poor survival, suggesting a potential role as a novel prognostic marker in breast cancer. Furthermore, molecular pathways regulated by mir-24-3p are potential therapeutic targets for metastatic breast cancer. 


\section{Data accession}

All the raw count tables for nanostring data and it's quality control tests areprovided in the supplementary data (Supplementary data 2 and 3). Theclinical metadata for the nanostring samples are provided in supplementary data 1. TCGA mir-24-3p expression profiles and their clinical information areprovided in supplementary data 4 .

\section{Author contributions}

Conception and design: A. Khodadadi-Jamayran, B. Akgoloksuz, Y. Afanasyeva, A. Zeleniuch-Jacquotte, A. Tsirigos, F. J. Esteva

Development of methodology: A. KhodadadiJamayran, B. Akgoloksuz, Y. Afanasyeva, A. Heguy, A. Zeleniuch-Jacquotte, A. Tsirigos, F. J. Esteva

Acquisition of data (provided animals, acquired and managed patients, provided facilities, etc.): Heguy, M. Thompson, K. Ray, X. Wu, D. Tripathy, F. J. Esteva

Analysis and interpretation of data (e.g., statistical analysis, biostatistics, computational analysis): A. Khodadadi-Jamayran, B. Akgoloksuz, Y. Afanasyeva, A. Heguy, M. Thompson, K. Ray, A. Giro-Perafita, I. Sanchez, B. Dynlacht, A. Zeleniuch-Jacquotte, A. Tsirigos, F. J. Esteva

Writing, review, and/or revision of the manuscript: A. Khodadadi-Jamayran, B. Akgoloksuz, Y. Afanasyeva, A. Heguy, M. Thompson, K. Ray, A. GiroPerafita, I. Sanchez, B. Dynlacht, X. Wu, D. Tripathy, A. Zeleniuch-Jacquotte, A. Tsirigos, F. J. Esteva

Administrative, technical, or material support (i.e., reporting or organizing data, constructing databases): Y. Afanasyeva, A. Heguy, M. Thompson, K. Ray, A. Zeleniuch-Jacquotte, A. Tsirigos, F. J. Esteva

Study supervision: A. Tsirigos, F. J. Esteva.

\section{ACKNOWLEDGMENTS}

We would like to thank the Departments of Breast Medical Oncology and Epidemiology at The University of Texas MD Anderson Cancer Center for their assistance in plasma procurement and annotated clinical data.

\section{CONFLICTS OF INTEREST}

The authors declare no potential conflicts of interest.

\section{FUNDING}

This study was supported by the Breast Cancer Research Foundation (FJE) and by the Center for Biospecimen Research and Development (CBRD) and the Genome Technology Center at the Perlmutter Cancer Center, NYU Langone Health (NIH/NCI 5P30CA016087 grant).

\section{REFERENCES}

1. Siegel RL, Miller KD, Jemal A. Cancer Statistics, 2017. CA Cancer J Clin. 2017; 67: 7-30.

2. Weigelt B, Peterse JL, van't Veer LJ. Breast cancer metastasis: markers and models. Nat Rev Cancer. 2005; 5: 591-602.

3. Adaniel C, Jhaveri K, Heguy A, Esteva FJ. Genome-based risk prediction for early stage breast cancer. Oncologist. 2014; 19: 1019-27.

4. Friedrichs K, Kugler G, Franke F, Terpe HJ, Arlt J, Regidor PA, Gunthert U. CD44 isoforms in prognosis of breast cancer. Lancet. 1995; 345: 1237.

5. Kwa M, Makris A, Esteva FJ. Clinical utility of geneexpression signatures in early stage breast cancer. Nat Rev Clin Oncol. 2017; 14:595-610.

6. Murthy RK, Varma A, Mishra P, Hess KR, Young E, Murray JL, Koenig KH, Moulder SL, Melhem-Bertrandt A, Giordano SH, Booser D, Valero V, Hortobagyi GN, et al. Effect of adjuvant/neoadjuvant trastuzumab on clinical outcomes in patients with HER2-positive metastatic breast cancer. Cancer. 2014; 120: 1932-8.

7. Brewster AM, Hortobagyi GN, Broglio KR, Kau SW, Santa-Maria CA, Arun B, Buzdar AU, Booser DJ, Valero V, Bondy M, Esteva FJ. Residual risk of breast cancer recurrence 5 years after adjuvant therapy. J Natl Cancer Inst. 2008; 100: 1179-83.

8. Esteva FJ, Sahin AA, Coombes K, Baker J, Cronin M, Walker M, Watson D, Cristofanilli M, Shak S, Hortobagi GN. Multi-gene RT-PCR assay for redicting recurrence in node negative breast cancer patients - M. D. Anderson Clinical Validation Study. Breast Cancer Res Treat. 2003; 82: A17.

9. Gaforio JJ, Serrano MJ, Sanchez-Rovira P, Sirvent A, Delgado-Rodriguez M, Campos M, de la Torre N, Algarra I, Duenas R, Lozano A. Detection of breast cancer cells in the peripheral blood is positively correlated with estrogenreceptor status and predicts for poor prognosis. Int J Cancer. 2003; 107: 984-90.

10. Su X, Malouf GG, Chen Y, Zhang J, Yao H, Valero V, Weinstein JN, Spano JP, Meric-Bernstam F, Khayat D, Esteva FJ. Comprehensive analysis of long non-coding RNAs in human breast cancer clinical subtypes. Oncotarget. 2014; 5:9864-76. https://doi.org/10.18632/oncotarget.2454.

11. Tavazoie SF, Alarcon C, Oskarsson T, Padua D, Wang Q, Bos PD, Gerald WL, Massague J. Endogenous human microRNAs that suppress breast cancer metastasis. Nature. 2008; 451: 147-52.

12. Manjili MH, Najarian K, Wang XY. Signatures of tumorimmune interactions as biomarkers for breast cancer prognosis. Future Oncol. 2012; 8: 703-11.

13. Li JY, Zhang Y, Zhang WH, Jia S, Kang Y, Tian R. Effects of differential distribution of microvessel density, possibly 
regulated by miR-374a, on breast cancer prognosis. Asian Pac J Cancer Prev. 2013; 14: 1715-20.

14. Jung EJ, Santarpia L, Kim J, Esteva FJ, Moretti E, Buzdar AU, Di Leo A, Le XF, Bast RC Jr, Park ST, Pusztai L, Calin GA. Plasma microRNA 210 levels correlate with sensitivity to trastuzumab and tumor presence in breast cancer patients. Cancer. 2012; 118: 2603-14.

15. Cancello G, Maisonneuve P, Rotmensz N, Viale G, Mastropasqua MG, Pruneri G, Montagna E, Dellapasqua S, Iorfida M, Cardillo A, Veronesi P, Luini A, Intra M, et al. Prognosis in women with small (T1mic,T1a,T1b) nodenegative operable breast cancer by immunohistochemically selected subtypes. Breast Cancer Res Treat. 2011; 127: 713-20.

16. Pei W, Dibekliouglu H, Tax DM, van der Maaten L. Multivariate Time-Series Classification Using the HiddenUnit Logistic Model. IEEE Trans Neural Netw Learn Syst. 2017; PP: 1-12.

17. Liu Y, Zhang Y, Li Q, Li J, Ma X, Xing J, Rong S, Wu Z, Tian Y, Li J, Jia L. MiRNAs Predict the Prognosis of Patients with Triple Negative Breast Cancer: A MetaAnalysis. PLoS One. 2017; 12: e0170088.

18. Sochor M, Basova P, Pesta M, Dusilkova N, Bartos J, Burda P, Pospisil V, Stopka T. Oncogenic microRNAs: miR-155, miR-19a, miR-181b, and miR-24 enable monitoring of early breast cancer in serum. BMC Cancer. 2014; 14: 448.

19. Kang H, Rho JG, Kim C, Tak H, Lee H, Ji E, Ahn S, Shin AR, Cho HI, Huh YH, Song WK, Kim W, Lee EK. The miR-24-3p/p130Cas: a novel axis regulating the migration and invasion of cancer cells. Sci Rep. 2017; 7: 44847.

20. Fiskaa T, Knutsen E, Nikolaisen MA, Jorgensen TE, Johansen SD, Perander M, Seternes OM. Distinct Small RNA Signatures in Extracellular Vesicles Derived from Breast Cancer Cell Lines. PLoS One. 2016; 11: e0161824.

21. Hannafon BN, Trigoso YD, Calloway CL, Zhao YD, Lum DH, Welm AL, Zhao ZJ, Blick KE, Dooley WC, Ding
WQ. Plasma exosome microRNAs are indicative of breast cancer. Breast Cancer Res. 2016; 18: 90.

22. Yu G, Jia Z, Dou Z. miR-24-3p regulates bladder cancer cell proliferation, migration, invasion and autophagy by targeting DEDD. Oncol Rep. 2017; 37: 1123-31.

23. Lu K, Wang J, Song Y, Zhao S, Liu H, Tang D, Pan B, Zhao H, Zhang Q. miRNA-24-3p promotes cell proliferation and inhibits apoptosis in human breast cancer by targeting p27Kip1. Oncol Rep. 2015; 34: 995-1002.

24. Du WW, Fang L, Li M, Yang X, Liang Y, Peng C, Qian W, O'Malley YQ, Askeland RW, Sugg SL, Qian J, Lin J, Jiang $Z$, et al. MicroRNA miR-24 enhances tumor invasion and metastasis by targeting PTPN9 and PTPRF to promote EGF signaling. J Cell Sci. 2013; 126: 1440-53.

25. Chen L, Luo L, Chen W, Xu HX, Chen F, Chen LZ, Zeng WT, Chen JS, Huang XH. MicroRNA-24 increases hepatocellular carcinoma cell metastasis and invasion by targeting p53: miR-24 targeted p53. Biomed Pharmacother. 2016; 84: 1113-8.

26. Cui S, Liao X, Ye C, Yin X, Liu M, Hong Y, Yu M, Liu Y, Liang H, Zhang CY, Chen X. ING5 suppresses breast cancer progression and is regulated by miR-24. Mol Cancer. 2017; $16: 89$.

27. Sasaki Y, Minamiya Y, Takahashi N, Nakagawa T, Katayose Y, Ito A, Saito H, Motoyama S, Ogawa J. REG1A expression is an independent factor predictive of poor prognosis in patients with breast cancer. Ann Surg Oncol. 2008; 15: 3244-51.

28. Yu G, Wang LG, Han Y, He QY. clusterProfiler: an R package for comparing biological themes among gene clusters. OMICS. 2012; 16: 284-7.

29. Krzywinski M, Schein J, Birol I, Connors J, Gascoyne R, Horsman D, Jones SJ, Marra MA. Circos: an information aesthetic for comparative genomics. Genome Res. 2009; 19: 1639-45. 